\title{
BMJ Open Pneumococcal colonisation density: a new marker for disease severity in HIV-infected adults with pneumonia
}

\author{
Werner C Albrich, ${ }^{1,2}$ Shabir A Madhi, ${ }^{2,3}$ Peter V Adrian, ${ }^{2}$ Nadia van Niekerk, ${ }^{2}$ \\ Jean-Noel Telles, ${ }^{4} \mathrm{~N}$ Ebrahim, ${ }^{2}$ Melina Messaoudi, ${ }^{4}$ Glaucia Paranhos-Baccalà, ${ }^{4}$ \\ Sven Giersdorf, ${ }^{5}$ Guy Vernet, ${ }^{4,6}$ Beat Mueller, ${ }^{7}$ Keith P Klugman ${ }^{2,8,9}$
}

To cite: Albrich WC, Madhi SA, Adrian PV, et al. Pneumococcal colonisation density: a new marker for disease severity in HIVinfected adults with pneumonia. BMJ Open 2014;4:e005953. doi:10.1136/bmjopen-2014005953

- Prepublication history and additional material is available. To view please visit the journal (http://dx.doi.org/ 10.1136/bmjopen-2014005953).

Received 23 June 2014 Accepted 24 July 2014

CrossMark

For numbered affiliations see end of article.

Correspondence to Dr Werner C Albrich; Werner.albrich@kssg.ch

\section{ABSTRACT}

Objective: A high genomic load of Pneumococcus from blood or cerebrospinal fluid has been associated with increased mortality. We aimed to analyse whether nasopharyngeal colonisation density in HIV-infected patients with community-acquired pneumonia (CAP) is associated with markers of disease severity or poor outcome.

Methods: Quantitative IytA real-time PCR was performed on nasopharyngeal swabs in HIV-infected South African adults hospitalised for acute CAP at Chris Hani Baragwanath Hospital, Soweto, South Africa. Pneumonia aetiology was considered pneumococcal if any sputum culture or Gram stain, urinary pneumococcal C-polysaccharide-based antigen, blood culture or whole blood lytA real-time PCR revealed pneumococci.

Results: There was a moderate correlation between the mean nasopharyngeal colonisation densities and increasing CURB65 scores among all-cause patients with pneumonia (Spearman correlation coefficient $r=0.15, p=0.06$ ) or with the Pitt bacteraemia score among patients with pneumococcal bacteraemia $(p=0.63)$. In patients with pneumococcal pneumonia, nasopharyngeal pneumococcal colonisation density was higher among non-survivors than survivors ( 7.7 vs 6.1 $\log _{10}$ copies $/ \mathrm{mL}$, respectively, $\mathrm{p}=0.02$ ) and among those who had pneumococci identified from blood cultures and/or by whole blood lytA real-time PCR than those with non-bacteraemic pneumococcal pneumonia (6.6 vs $5.6 \log _{10}$ copies/mL, $p=0.03$ ). Nasopharyngeal colonisation density correlated positively with the biomarkers procalcitonin (Spearman correlation coefficient $r=0.37, p<0.0001$ ), proadrenomedullin $(r=0.39, p=0.008)$ and copeptin $(r=0.30, p=0.01)$. Conclusions: In addition to its previously reported role as a diagnostic tool for pneumococcal pneumonia, quantitative nasopharyngeal colonisation density also correlates with mortality and prognostic biomarkers. It may also be useful as a severity marker for pneumococcal pneumonia in HIV-infected adults.

\section{INTRODUCTION}

Community-acquired pneumonia (CAP) and influenza are the second leading natural

\section{Strengths and limitations of this study}

- This study shows, for the first time, that nasopharyngeal (NP) colonisation density of Streptococcus pneumoniae is a prognostic marker in HIV-infected adults with pneumonia.

- NP density, as measured with a lytA real-time PCR, correlated with survival, bacteraemia and prognostic biomarkers.

- This information might be useful for clinical management decisions relating to site of care and antibiotic choice and duration.

- A major limitation is that it was tested in a single well-characterised cohort of HIV-infected South African adults hospitalised for pneumonia, whereas it needs to be confirmed in other settings and in HIV-uninfected adults.

- Other limitations were the lack of arterial blood gas analysis, which prevented us from calculating the Pneumonia Severity Index; as well as the small number of patients admitted to an intensive care unit.

cause of death in South Africa, responsible for $6.6-7.5 \%$ of deaths between 2009 and 2011. ${ }^{1}$ Streptococcus pneumoniae is the most common aetiology of CAP in adults regardless of setting and clinical severity. ${ }^{2}$ This is also the case for HIV-infected adults and has been confirmed in various aetiological studies from Africa. $^{3-8}$ In HIV-infected adults, Pneumococcus has been estimated to be implicated in $20 \%$ of bacterial pneumonias, $40 \%$ of pneumonias with an identified organism and $70 \%$ of pneumonias with positive blood cultures. ${ }^{9}$ Pneumococcal pneumonia carries a mortality of about $10-15 \%$ with slightly higher mortality in patients with bacteraemic pneumococcal pneumonia, ${ }^{10-14}$ which has been roughly unchanged since the advent of antibiotic therapy. ${ }^{15}$ Outcomes are worse for HIV-infected patients with pneumococcal pneumonia in some studies ${ }^{5}$ but not others. ${ }^{9}$ 
Information on prognosis is useful to assign limited medical resources including treatment and site-of-care decisions or to intensify therapy. The prognosis for patients with pneumonia is a complex interplay between host and microbiological factors. Current prognostic scoring systems such as the CURB65 score $^{16}$ or the Pneumonia Severity Index (PSI) ${ }^{17}$ are based on underlying conditions, clinical and laboratory characteristics. Interestingly, these scores have not been validated in patients with HIV infection even though HIV is a recognised risk factor for poor outcome in patients with pneumococcal pneumonia. ${ }^{5}$ Strikingly, these scores also do not take into account microbiological data. Novel biomarkers are increasingly used to provide prognostic information in patients with pneumonia, correlate with clinical severity scores ${ }^{18}$ and might provide additional information beyond clinical scores. ${ }^{19}$

Since organism burden has been difficult to assess in bacterial infections prior to more widespread use of quantitative molecular techniques, relatively little information is available on its prognostic impact. A high genomic load of Pneumococcus from cerebrospinal fluid and blood, respectively, has been associated with poor outcome and increased mortality in Malawian children with pneumococcal meningitis ${ }^{20}$ and Malawian children and European adults with bacteraemic pneumococcal pneumonia. ${ }^{13} 2021$ Non-invasive specimens would be preferable due to ease of sample collection and due to low diagnostic yields of blood specimens. We recently proposed quantitative nasopharyngeal (NP) colonisation density as a novel diagnostic tool for pneumococcal aetiology in HIV-infected adults with pneumonia. ${ }^{22}$ In this study, we aimed to analyse whether NP colonisation density is associated with clinical and biological markers of disease severity or poor outcome in the same cohort of patients with pneumonia.

\section{METHODS}

Details of the analysed patients were previously described. ${ }^{22}$ In brief, we enrolled adults (age $\geq 18$ years) who were hospitalised at Chris Hani Baragwanath Hospital, Soweto, South Africa, with acute X-ray confirmed pneumonia CAP. Active tuberculosis or current antituberculous treatment at diagnosis were exclusion criteria for enrolment, whereas patients with current or recent antibiotic therapy were not excluded. S. pneumoniae was identified by colony morphology, optochin susceptibility and bile solubility. Urine was tested with the immunochromatographic BinaxNow S. pneumoniae test (ICT) for pneumococcal C-polysaccharide according to the manufacturer's instructions. Quantitative lytA realtime (rt) PCR was performed on NP swab as described. ${ }^{22}$ For whole blood testing, nucleic acids were extracted from $200 \mu \mathrm{L}$ of clinical samples using the QIAamp DNA Blood Mini Kit (Qiagen, the Netherlands) and eluted in $100 \mu \mathrm{L}$ of elution buffer. $5 \mu \mathrm{L}$ of extracted DNA was subjected to a triplex rtPCR for Pneumococcus (lytA),
Haemophilus influenzae type b, and Staphylococcus aureus using the iQ Multiplex Powermix (Bio-Rad, USA). Owing to the lack of a diagnostic gold standard, pneumococcal pneumonia (SP-CAP) was considered present if a composite diagnostic standard was positive in patients with CAP, that is, if pneumococci were detected by either blood culture, urine ICT, good quality (ie, $>25$ neutrophils and $<10$ epithelial cells per high-power field) sputum Gram stain or culture, or whole blood lytA rtPCR. In an expanded composite diagnostic (eSP-CAP) we included a quantitative NP density $>8000$ copies $/ \mathrm{mL}^{22}$ as an additional criterion for pneumococcal pneumonia. Antimicrobial activity in urine as evidence of previous antimicrobial use was measured as previously described. ${ }^{22}$

HIV counselling and testing were offered to all patients with unknown or negative HIV serostatus. Testing was performed using the Architect HIV Ag/Ab Combo assay (Abbott) and, if positive, was confirmed by Elecsys HIV combi assay (Elecsys 2010 analyzer, Roche). The main study population included only patients who were already known to be HIV-infected or newly diagnosed with HIV infection.

The CURB65 score was calculated as according to Lim et al. ${ }^{16}$ For patients with bacteraemic pneumonia, that is, positive blood culture or whole blood lytA rtPCR, we calculated the Pitt bacteraemia score. ${ }^{23}{ }^{24}$ Infection-related and prognosis-related biomarkers were measured in batch with a highly sensitive research assay for procalcitonin (BRAHMS PCT LIA sensitive) and commercial assays for mid-regional proadrenomedullin (BRAHMS MR-proADM KRYPTOR), mid-regional proatrial natriuretic peptide (BRAHMS MR-proANP KRYPTOR) and copeptin (BRAHMS Copeptin KRYPTOR, all Thermo Scientific Biomarkers, Hennigsdorf, Germany) after storage at $-70^{\circ} \mathrm{C}$. C reactive protein (CRP) was measured immediately on site by the routine diagnostics laboratory (CRP immunoturbidometry, 717 automated analyzer; Boehringer Mannheim/Hitachi, Mannheim, Germany) if requested by the treating physicians.

Continuous variables were compared with two-sided pooled $\mathrm{t}$ tests or a Mann-Whitney-Wilcoxon test, as appropriate, and proportions calculated with Pearson's $\chi^{2}$ test or Fisher's exact test, as appropriate. For relationship between continuous variables, we used Spearman correlation coefficients. One-way analysis of variance (ANOVA) and Kruskal-Wallis tests were applied for comparisons of continuous dependent variables between more than two categorical independent variables, as appropriate. Univariate and multivariate analyses were performed to identify predictors of hospital mortality. Variables significant in the univariate analysis and those of special interest were included in the multivariate analysis. The area under the receiver operating characteristic curve (AUC-ROC) was calculated as indicator for diagnostic accuracy to predict hospital mortality. $p$ Values $\leq 0.05$ were considered significant.

All patients and controls provided written informed consent. 


\section{RESULTS}

Of 514 patients with acute clinical pneumonia, 370 had X-ray confirmed pneumonia (CAP). Analyses were restricted to those 280 patients with CAP in whom HIV-infection was present or newly diagnosed. Based on the composite diagnostic criteria, SP-CAP was diagnosed in $99(35.4 \%)$ of 280 patients: 75 had either a positive urine ICT or blood culture, or good quality (ie, $>25$ neutrophils and $<10$ epithelial cells per high-power field) sputum Gram stain or culture with pneumococcus ${ }^{22} ; 58$ patients had a positive whole blood lytA rtPCR. A lytA rtPCR from NP $>8000$ copies $/ \mathrm{mL}$ was present in 126 patients, resulting in a diagnosis of pneumococcal CAP based on the expanded composite diagnostic in 150 (53.6\%) of 280 patients. The in-hospital case death rate was not different between those with (11.7\%) and those without $(14.1 \%$; $\mathrm{p}=0.53)$ pneumococcal aetiology.

Mean pneumococcal colonisation density was not significantly different between patients with and those without antimicrobial activity in urine $(3.36(95 \%$ CI 2.82 to 3.89 ) copies/mL vs 3.95 (95\% CI 3.30 to 4.60 ); $\mathrm{p}=0.16$ ). There was also no difference in in-hospital mortality between those with and those without antimicrobial activity in urine $(13.2 \%$ vs $11.5 \%$; $\mathrm{p}=0.69)$.

\section{Correlation between colonisation density and clinical scores}

As a prognostic marker for mortality, we correlated NP colonisation with the CURB65 score. Colonisation density measured by lyt $A$ rtPCR did not significantly correlate with increasing CURB65 classes either among cases with CAP (Spearman correlation coefficient $\mathrm{r}=0.15, \mathrm{p}=0.06$ ) or among cases with SP-CAP (Spearman correlation coefficient $\mathrm{r}=0.14, \mathrm{p}=0.19)$. The mean colonisation density of cases with bacteraemic SP-CAP did not vary significantly for different Pitt bacteraemia scores $(0$ : $6.5 \log _{10}$ copies/mL, 1: $6.8 \log _{10}$ copies/mL; 2: $7.0 \log _{10}$
copies/mL; 3: $4.8 \log _{10}$ copies/mL; p=0.63). Hospital mortality did not vary significantly between CURB65 scores $(0: 13.1 \%$; 1: 11.1\%; 2: $11.3 \%$; 3: $25.0 \%$; $4: 0 \%$; $\mathrm{p}=0.56)$ or-among those with bacteraemic pneumococcal pneumonia-between Pitt bacteraemia score points (0: 20.7\%; 1: 10.7\%; 2: 16.7\%; 3: 0\%).

\section{Correlation between colonisation density and bacteraemia}

Presence of pneumococci in blood (by culture or whole blood lytA rtPCR) among cases with SP-CAP was associated with a higher pneumococcal NP colonisation density than if SP-CAP was non-bacteraemic (6.6 vs 5.6 $\log _{10}$ copies $/ \mathrm{mL}, \mathrm{p}=0.03$; figure 1 ). Similarly, growth of $S$. pneumoniae from blood cultures was associated with higher NP colonisation density among patients with SP-CAP than those without ( 7.1 vs $6.1 \log _{10}$ copies $/ \mathrm{mL}$, $\mathrm{p}=0.04)$.

\section{Correlation between colonisation density and biomarkers}

The levels of the biomarkers procalcitonin, proadrenomedullin and CRP, but not of copeptin and ProANP were significantly higher for patients with SP-CAP than for patients with CAP without pneumococcal aetiology (table 1).

There was a positive correlation between increasing NP colonisation density among those colonised with Pneumococcus and increasing procalcitonin values in cases with CAP (Spearman correlation coefficient $\mathrm{r}=0.37, \mathrm{p}<0.0001)$ and in cases with SP-CAP $(\mathrm{r}=0.37$, $\mathrm{p}=0.001$; figure $2 \mathrm{~A}$ ).

Similarly, there was a significant positive correlation between increasing NP colonisation density among those colonised with Pneumococcus and increasing proadrenomedullin values in cases with CAP $(\mathrm{r}=0.34$, $\mathrm{p}<0.0001)$ and in cases with SP-CAP $(\mathrm{r}=0.40, \mathrm{p}=0.008$; figure 2B). A similar positive correlation was observed between increasing copeptin and increasing NP

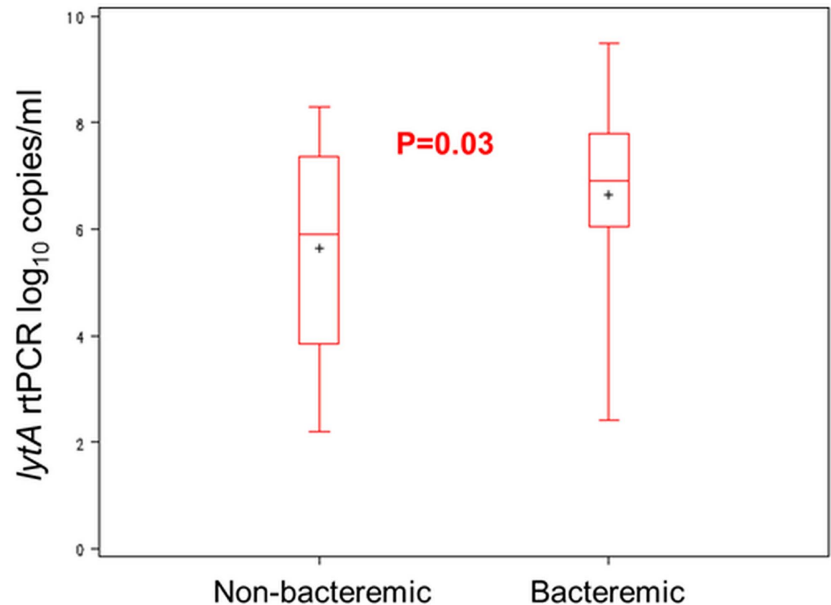

Non-bacteremic

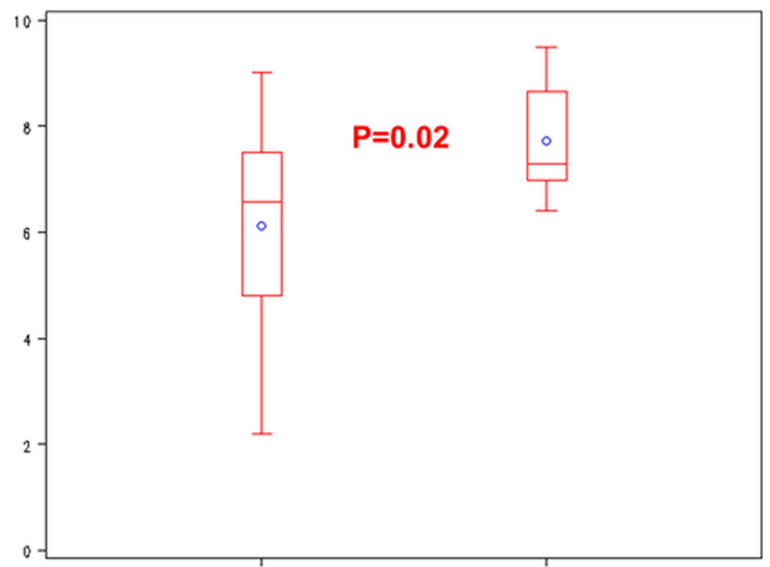

Hospital-survivor Hospital-non-survivor

Figure 1 Association of nasopharyngeal colonisation density with bacteraemia and death. Nasopharyngeal colonisation density according to bacteraemia (either positive blood cultures or positive lytA real-time (rt) PCR from whole blood) and survival in HIV-infected patients with pneumococcal pneumonia. $+{ }^{\circ}$ represent the mean; length of the box represents the IQR between the 25th and 75th centiles; horizontal line in the box represents median; whiskers represent minimum and maximum values. 
Table 1 Biomarkers in HIV-infected patients with community-acquired pneumonia

\begin{tabular}{|c|c|c|c|c|c|c|}
\hline & \multicolumn{3}{|c|}{ Based on composite diagnostic standard* } & \multicolumn{3}{|c|}{$\begin{array}{l}\text { Based on expanded composite diagnostic } \\
\text { standard } \dagger\end{array}$} \\
\hline & $\begin{array}{l}\text { Pneumococcal } \\
\text { aetiology* }\end{array}$ & $\begin{array}{l}\text { Non-pneumococcal } \\
\text { aetiology* }\end{array}$ & p Value* & $\begin{array}{l}\text { Pneumococcal } \\
\text { aetiology }\end{array}$ & $\begin{array}{l}\text { Non-pneumococcal } \\
\text { aetiologyt }\end{array}$ & p Valuet \\
\hline rocalcitc & ) & ) & -0.000 & 16 & 5. & ( \\
\hline $\begin{array}{l}\text { roadrenomedullin } \\
\text { AR-proADM; nmol/L) }\end{array}$ & $2.9(n=80)$ & $1.9(n=$ & 0.01 & 2.8( & $1.6(r$ & 0.003 \\
\hline $\begin{array}{l}\text { roatrial natriuretic } \\
\text { eptide (MR-proANP; } \\
\text { nol/L) }\end{array}$ & $119.4(n=81)$ & $120.9(n=156)$ & 0.93 & $118.7(n=126)$ & $122.3(n=111)$ & 0.84 \\
\hline omol/L) & 73. & 46 & 0 & 68 & & \\
\hline CRP (mg/L) & $282.9(n=20)$ & $134.4(\mathrm{n}=27)$ & $<0.0001$ & $248.2(n=27)$ & $129.3(n=20)$ & 0.0009 \\
\hline \multicolumn{7}{|c|}{$\begin{array}{l}\text { Since } C \text { reactive protein (CRP) values were available only when requested by the treating physicians, we compared patients with available } \\
\text { values for CRP to patients without available CRP values. There was no difference in mean age, in mean nasopharyngeal colonisation density } \\
\text { and no difference in pneumococcal diagnosis. However, patients with an available CRP value had a significantly higher ( } 30.6 \% \text { ) in-hospital } \\
\text { mortality compared to patients without a CRP value (15.3\%) ( } \mathrm{p}=0.02) \text {. } \\
{ }^{*} \text { Pneumococcal and non-pneumococcal aetiology were defined according to composite diagnostic standard (pneumococci were detected by } \\
\text { either blood culture, urine immunochromatographic BinaxNow Streptococcus pneumoniae test, good quality (ie, }>25 \text { neutrophils and }<10 \\
\text { epithelial cells per high-power field) sputum Gram stain or culture, or whole blood lytA rtPCR). (rt, real-time). } \\
\text { †Pneumococcal and non-pneumococcal aetiology were defined according to composite diagnostic standard (pneumococci were detected by } \\
\text { either blood culture, urine immunochromatographic BinaxNow Streptococcus pneumoniae test, good quality (ie, }>25 \text { neutrophils and }<10 \\
\text { epithelial cells per high-power field) sputum Gram stain or culture, or whole blood lytA rtPCR or lytA rtPCR from nasal swab }>8000 \mathrm{copies} / \mathrm{mL} \text { ). }\end{array}$} \\
\hline
\end{tabular}

colonisation density among those colonised with Pneumococcus and in patients with CAP ( $\mathrm{r}=0.18, \mathrm{p}=0.03)$ and in patients with SP-CAP ( $\mathrm{r}=0.30, \mathrm{p}=0.01$; figure $2 \mathrm{C}$ ). There was a trend for a positive correlation between increasing NP colonisation density among those colonised with Pneumococcus and increasing ProANP values in patients with CAP $(\mathrm{r}=0.17, \mathrm{p}=0.047)$, but less so in patients with SP-CAP $(r=0.12, p=0.30$; figure 2D). In contrast, there was no significant correlation between increasing NP colonisation density and CRP values for patients with CAP $(r=0.13, p=0.51)$ or for patients with $\mathrm{SP}-\mathrm{CAP}(\mathrm{r}=0.35, \mathrm{p}=0.17$; figure $2 \mathrm{E})$.

\section{Correlation between colonisation density and in-hospital mortality}

Among patients with SP-CAP who had pneumococcal colonisation in the nasopharynx, mean NP colonisation density was significantly higher in those who died $(n=8$; $7.72 \log _{10}$ copies $\left./ \mathrm{mL}\right)$ than those who survived $(n=76$; $6.11 \log _{10}$ copies/mL; $\mathrm{p}=0.02$ ). All non-survivors had very high NP colonisation densities, which were at least $6.4 \log _{10}$ copies $/ \mathrm{mL}$ and thus more than $2.5 \log _{10}$ copies/mL higher than the cut-off of $3.9 \log _{10}$ copies/ $\mathrm{mL}$ proposed to diagnose pneumococcal aetiology (figure 1).

In univariate analysis the levels of copeptin, ProANP, proadrenomedullin and procalcitonin and the NP pneumococcal colonisation density were significantly associated with hospital mortality in patients with CAP (table 2). In multivariable logistic regression, proadrenomedullin (aOR 1.196, 95\% CI 1.011 to $1.414 ; \mathrm{p}=0.04$ ) and quantitative $l y t A$ rtPCR from NP (aOR 1.870; 95\% CI 1.047 to $3.342 ; \mathrm{p}=0.03$ ) were independent predictors of hospital mortality (table 2).
In patients with SP-CAP, receipt of highly active antiretroviral therapy (HAART) and increasing levels of the biomarkers copeptin, ProANP, proadrenomedullin and increasing NP pneumococcal colonisation density were associated with hospital mortality (table 2). In a multivariable logistic regression model, there were trends for increasing age (aOR 1.09; 95\% CI 0.99 to $1.20 ; \mathrm{p}=0.10$ ) and quantitative $l y t A$ rtPCR from NP (aOR 2.10; $95 \%$ CI 0.95 to $4.65 ; \mathrm{p}=0.07$ ) as predictors of mortality (table 2 ).

The prognostic accuracy as determined by the ROC-AUC curves showed the best prediction of hospital mortality from all-cause pneumonia for the prognostic biomarkers proadrenomedullin, ProANP and copeptin, with lower AUC observed for quantitative lytA rtPCR from NP (see online supplementary table S3, figure 3A). In patients with SP-CAP, proadrenomedullin and quantitative lytA rtPCR from NP had the highest AUC (0.77 and 0.74 , respectively, figure $3 \mathrm{~B}$ ).

\section{DISCUSSION}

This study shows, for the first time, that NP colonisation density as measured by quantitative lytA rtPCR was predictive of bacteraemia and mortality in HIV-infected South African adults with CAP. Colonisation density correlated moderately with prognostic biomarkers indicative of poor outcome.

Despite the theoretical limitation being that molecular diagnostics cannot distinguish between dead and alive bacteria, we previously reported a good correlation between quantitative $l y t A$ rtPCR and quantitative NP cultures. $^{22}$ A pneumococcal colonisation density cut-off $>8000$ copies / $\mathrm{mL}$ for HIV-infected adults with CAP was a highly sensitive and specific diagnostic tool for pneumococcal aetiology. ${ }^{22}$ This supported the pathophysiological 
A

lytA rtPCR $\log _{10}$ copies $/ \mathrm{ml}$

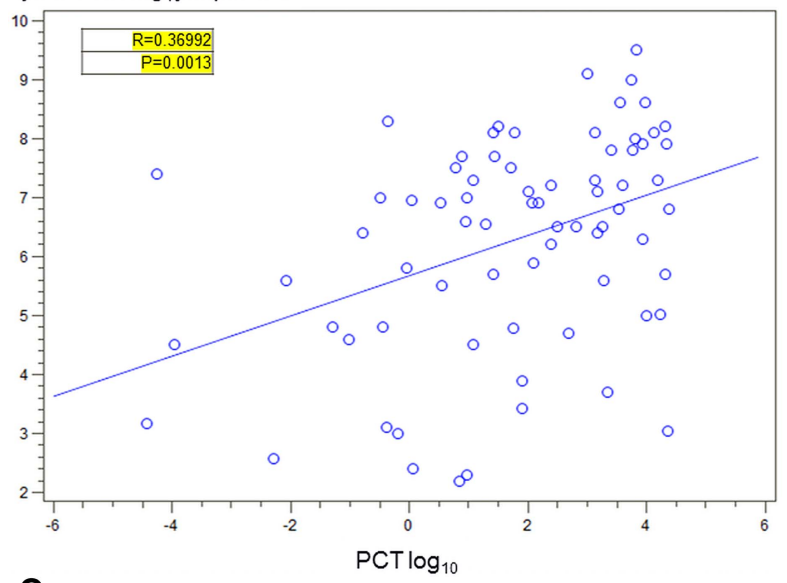

C

lytA rtPCR $\log _{10}$ copies $/ \mathrm{ml}$

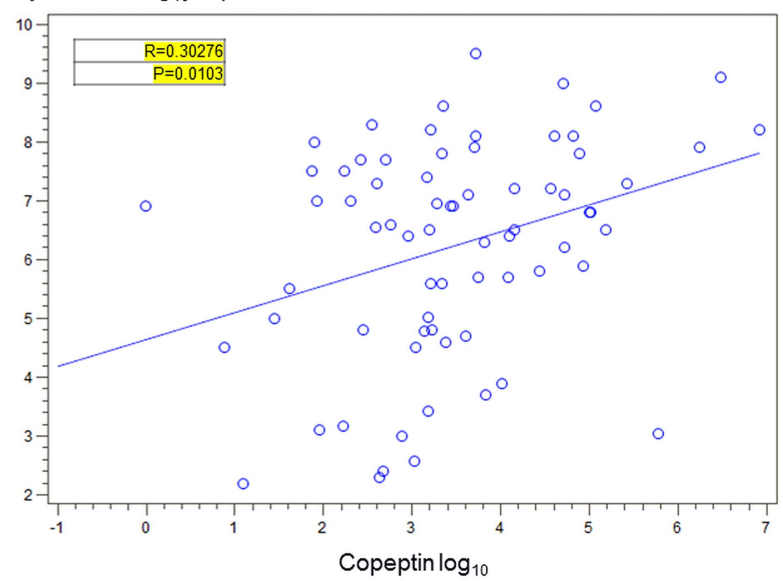

B

IytA rtPCR $\log _{10}$ copies/ml

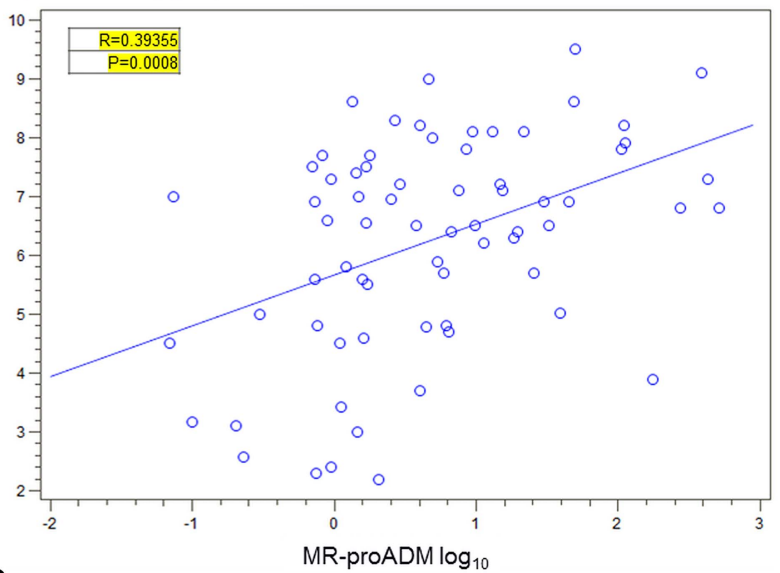

IytA rtPCR $\log _{10}$ copies $/ \mathrm{ml}$

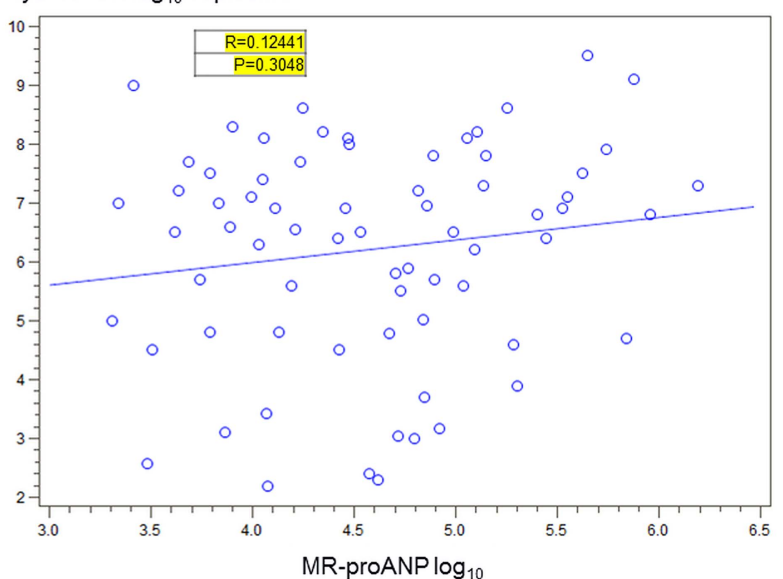

E IytA rtPCR $\log _{10}$ copies $/ \mathrm{ml}$

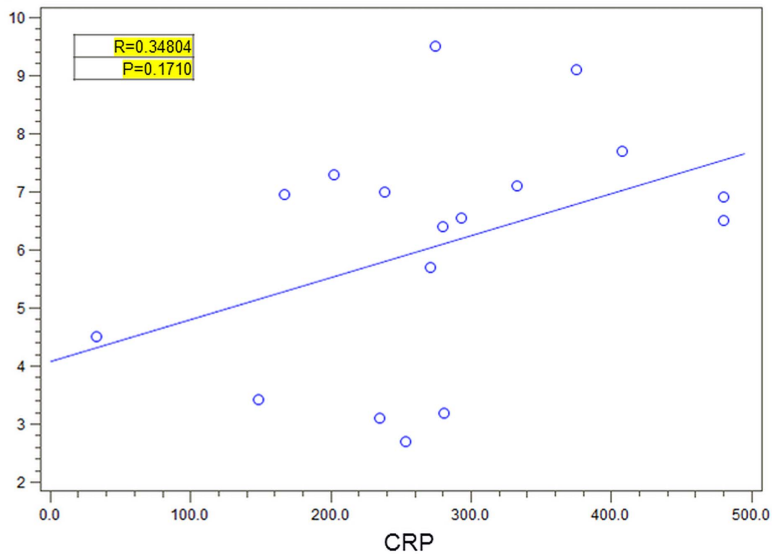

Figure 2 Correlation between nasopharyngeal colonisation density and biomarkers in pneumococcal pneumonia.

(A) Procalcitonin. (B) MR-proADM. (C) Copeptin. (D) MR-proANP. (E) C reactive protein. Correlation between nasopharyngeal colonisation density and biomarkers among HIV-infected cases with SP-CAP who are colonised with pneumococcus.

Pneumococcal aetiology (SP-CAP) in patients with CAP was defined as pneumococcal detection from either blood culture, urine immunochromatographic BinaxNow Streptococcus pneumoniae test, good quality (ie, $>25$ neutrophils and $<10$ epithelial cells per high-power field) sputum Gram stain or culture, or whole blood lytA real-time (rt) PCR. Counts are depicted after logarithmic transformation.

concept of a critical pneumococcal colonisation density in the nasopharynx, at which the risk of pneumococcal microaspiration and subsequent lower respiratory tract infection increase. ${ }^{25}$ Owing to the lack of lung specimens or bronchoalveolar lavage samples representing the direct site of infection, we instead chose to correlate NP 
Table 2 Predictors of in-hospital mortality in HIV-infected adults with CAP and SP-CAP

\begin{tabular}{|c|c|c|c|c|c|c|c|c|c|c|c|c|}
\hline & \multicolumn{6}{|l|}{ CAP } & \multicolumn{6}{|c|}{ SP-CAP } \\
\hline & \multicolumn{3}{|c|}{ Univariate analysis } & \multicolumn{3}{|c|}{ Multivariable analysis } & \multicolumn{3}{|c|}{ Univariate analysis } & \multicolumn{3}{|c|}{ Multivariable analysis } \\
\hline & OR & $\begin{array}{l}95 \% \\
\text { Confidence } \\
\text { limit }\end{array}$ & p Value & OR & $\begin{array}{l}95 \% \\
\text { Confidence } \\
\text { limit }\end{array}$ & p Value & OR & $\begin{array}{l}95 \% \\
\text { Confidence } \\
\text { limit } \\
\end{array}$ & p Value & OR & $\begin{array}{l}95 \% \\
\text { Confidence } \\
\text { limit }\end{array}$ & p Value \\
\hline $\begin{array}{l}\text { Pneumococcal aetiology } \\
\text { (vs not) }\end{array}$ & 0.806 & $0.378-1.719$ & 0.5766 & & & & & & & & & \\
\hline $\begin{array}{l}\text { Bacteraemic } \\
\text { pneumococcal } \\
\text { pneumonia (vs not) }\end{array}$ & 1.289 & $0.585-2.841$ & 0.5285 & & & & 5.37 & $0.655-44.055$ & 0.12 & & & \\
\hline Male (vs female) & 1.083 & $0.527-2.225$ & 0.8292 & & & & 0.91 & $0.247-3.36$ & 0.88 & & & \\
\hline Age & 1.028 & $0.992-1.065$ & 0.1307 & & & & 1.04 & $0.97-1.108$ & 0.29 & 1.086 & $0.985-1.198$ & 0.097 \\
\hline $\begin{array}{l}\text { Cotrimoxazole } \\
\text { prophylaxis (vs not) }\end{array}$ & 0.211 & $0.028-1.603$ & 0.1327 & & & & 1.08 & $0.121-9.765$ & 0.94 & & & \\
\hline CD4 count & 0.999 & $0.996-1.002$ & 0.4752 & & & & 0.998 & $0.992-1.005$ & 0.58 & & & \\
\hline HAART (vs not) & 0.838 & $0.238-2.948$ & 0.783 & & & & 5.85 & $1.175-29.144$ & 0.03 & & & \\
\hline CURB65 & 1.137 & $0.782-1.654$ & 0.5007 & & & & 1.425 & $0.684-2.97$ & 0.34 & & & \\
\hline Copeptin & 1.005 & $1.002-1.008$ & 0.0013 & & & & 1.005 & $1.001-1.009$ & 0.0254 & & & \\
\hline MR-proANP & 1.005 & $1.003-1.008$ & $<0.0001$ & & & & 1.008 & $1.001-1.014$ & 0.0156 & & & \\
\hline PCT & 1.013 & $0.996-1.030$ & 0.1226 & & & & 1.018 & $0.993-1.043$ & 0.1597 & & & \\
\hline MR-proADM & 1.260 & $1.123-1.412$ & $<0.0001$ & 1.196 & $1.011-1.414$ & 0.0366 & 1.266 & $1.064-1.505$ & 0.0076 & & & \\
\hline $\log _{10} / y t A$ rtPCR NP & 1.284 & $0.985-1.672$ & 0.0642 & 1.870 & $1.047-3.342$ & 0.0345 & 2.262 & $1.086-4.712$ & 0.03 & 2.104 & $0.952-4.65$ & 0.0659 \\
\hline
\end{tabular}

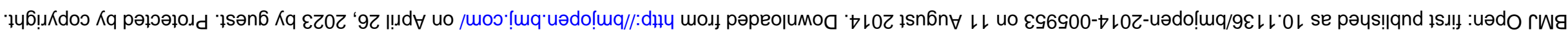


A

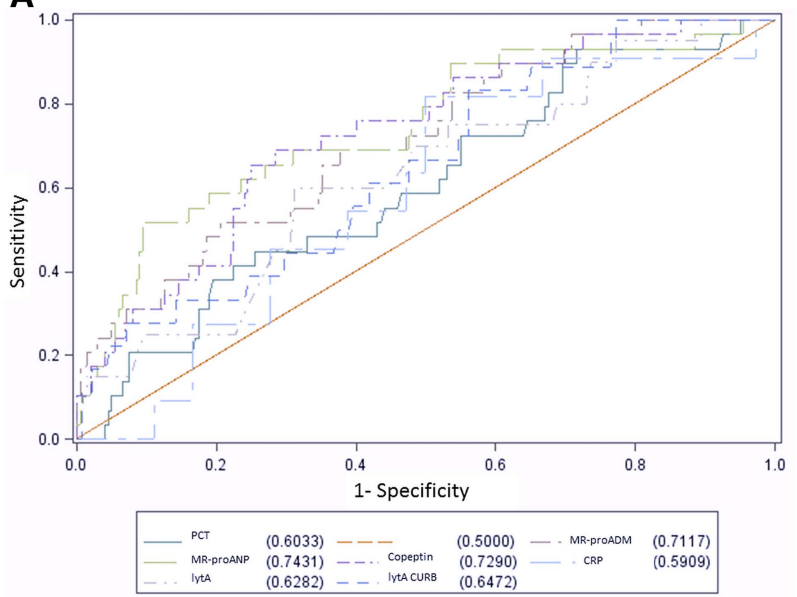

B

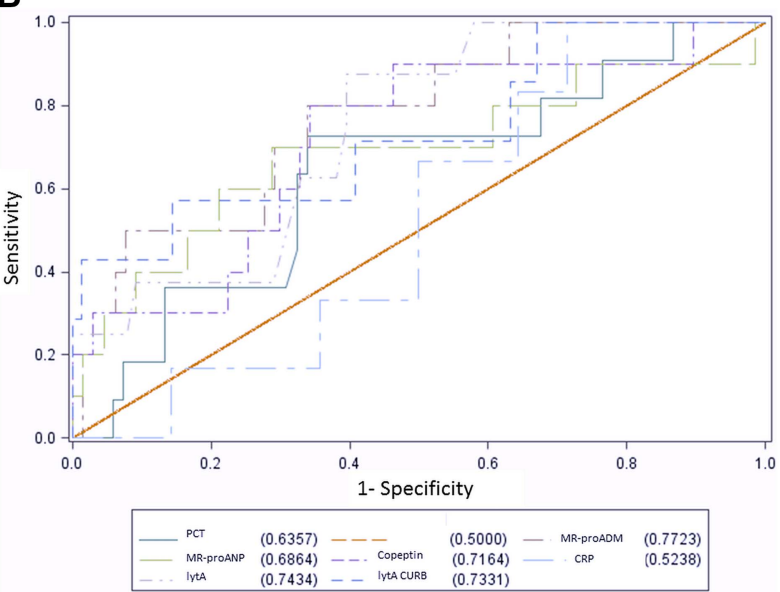

Figure 3 Prognostic accuracy for hospital mortality. (A) All-cause pneumonia. (B) Pneumococcal pneumonia. Receiver operating characteristic curves with area under the curve for individual parameters. Pneumococcal aetiology defined as pneumococcal detection from either blood culture, urine immunochromatographic BinaxNow Streptococcus pneumoniae test, good quality (ie, $>25$ neutrophils and $<10$ epithelial cells per high-power field) sputum Gram stain or culture, or whole blood lyt $A$ real-time (rt) PCR.

colonisation density with prognosis. Despite the biological plausibility that clinical severity and risk of poor outcome correlate with a higher organism density at the site of infection, there are surprisingly few data to support this hypothesis in patients with pneumonia. Major reasons might be the difficulty of measuring the organism burden prior to the wider application of quantitative molecular methods and obtaining the appropriate specimen for this measurement. Studies in the first half of the 20th century using quantitative blood cultures reported a dose-effect relationship between organism burden and mortality. ${ }^{26}{ }^{27}$ As a surrogate marker for bacterial load in children with bacteraemic SP-CAP in Spain, a shorter time to positivity of blood cultures correlated with risk of hospitalisation, admission to the intensive care unit (ICU), meningitis or other non-pneumonic sources of bacteraemia and with a higher number of positive blood cultures. ${ }^{28}$ In children with pneumococcal meningitis in Malawi, higher DNA loads as measured with a lytA rtPCR from cerebrospinal fluid and blood were found in more non-survivors than survivors $(8.2 \mathrm{vs}$ $7.5 \log _{10}$ copies/mL, $\mathrm{p}=0.03$; and 3.8 vs $3.2 \log _{10}$ copies/ $\mathrm{mL}, \mathrm{p}=0.02$; respectively). All children with SP-CAP survived and had lower DNA loads $\left(0.42 \log _{10}\right.$ copies $\left./ \mathrm{mL}\right)$ than meningitis survivors and non-survivors. ${ }^{20}$ Genomic loads were higher for HIV-infected children $(n=59)$ than HIV-uninfected children ( $\mathrm{n}=39) \quad(\mathrm{p}=0.04$ for CSF; $\mathrm{p}=0.6$ for blood). In addition, blood bacterial DNA load correlated with plasma cytokines such as tumor necrosis factor- $\alpha$, interleukin (IL)-1 $\beta$, IL-6 and IL- $10 .{ }^{20}$ In a study of 45 Dutch adults with CAP, quantitative pneumococcal load measured with lytA PCR from whole blood was higher in patients with bacteraemia and in those who fulfilled criteria of the systemic inflammatory response syndrome. It correlated positively with markers for severity of disease including length of stay, CRP levels and mental status changes. ${ }^{21}$ In a Spanish study of 93 adults with pneumococcal CAP whole blood lytA copies independently predicted septic shock and receipt of mechanical ventilation. ${ }^{13}$ High lytA copy numbers $\left(\geq 10^{3} / \mathrm{mL}\right)$ were risk factors for bacteraemia $(\mathrm{OR}=6.3)$, septic shock $(\mathrm{OR}=8.0)$, acute kidney injury $(\mathrm{OR}=7.0)$, mechanical ventilation $(\mathrm{OR}=10.5)$, acute respiratory distress syndrome $(\mathrm{OR}=14.8)$, rapid radiological spread $(\mathrm{OR}=22.8)$ and mortality $(\mathrm{OR}=5.4) .{ }^{13} \mathrm{~A}$ recent study showed that HIV and influenza infections were associated with higher lyt $A$ load in blood among South African patients with acute lower respiratory tract infections and demonstrated 3.6-fold increased mortality if pneumococcal load was $\geq 10^{5} / \mathrm{mL}$. ${ }^{29}$ Pleural fluid genomic pneumococcal load measured with ply rtPCR, which is a less specific target than $l y t A,{ }^{30}$ in children with SP-CAP correlated with positive cultures, higher biomarkers such as CRP and neutrophils and with a longer length of hospital stay. ${ }^{31}$ In another study, high blood lytA copy numbers in children $\leq 5$ years of age with CAP were predictive of a parapneumonic effusion. Children with empyema had higher values than those with simple parapneumonic effusion. Serotype 19A was associated with high bacterial loads as well as parapneumonic effusion or empyema. ${ }^{32}$

A limitation of applying quantitative molecular methods to invasive specimens in these studies is that only the relatively small subset of pneumococcal disease with documented invasiveness can be evaluated: approximately $10-25 \%$ of SP-CAP in adults ${ }^{33} 34$ and $<10 \%$ in children $^{3536}$ are bacteraemic. Therefore, application of quantitative molecular methods in NP swabs not only improves ease of specimen collection but also markedly increases the number of patients who could be assessed in this way. In this regard NP swabs are also superior to sputum, which frequently cannot be obtained in sufficient quantity and quality. ${ }^{37}$ In adults from New Zealand 
with CAP, the pneumococcal copy numbers measured by quantitative lytA rtPCR were higher in sputum than in serum or urine. ${ }^{38}$ However, sputum could only be analysed in 196 of 304 patients. The pneumococcal load in serum was associated with CURBage score, PSI, length of hospital stay and ICU admission, while increasing sputum load was only associated with severity of disease as measured with the CURBage score. ${ }^{38}$

To the best of our knowledge, ours is the first study that formally correlated quantitative NP pneumococcal load with clinical severity markers. Higher pneumococcal loads were detected in more bacteraemic than nonbacteraemic patients, thereby confirming previous studies, which used quantitative blood $^{13}$ or sputum ${ }^{38}$ loads. In addition, pneumococcal colonisation density tended to increase with increasing CURB65 scores, with significant albeit only moderate correlation. However, in this study, the CURB65 did not correlate with mortality. Data on the utility of the CURB65 score in HIV is largely absent. In the only prior study formally evaluating the CURB65 score it underestimated mortality and the requirement for ICU admission as HIV-infected patients had more than twice as high mortality and twice as long hospital and ICU stays compared with HIV-uninfected patients despite similar CURB65 scores. ${ }^{39}$ While reasons for this discrepant performance of the CURB65 score between HIV-infected and HIV-uninfected adults are not clear, the age factor seems less important in typically younger HIV-infected patients, whose biological ages are reportedly 10-14 years advanced. ${ }^{40-42}$ There are two possible nonexclusive explanations for the lack of correlation between NP colonisation density and the Pitt bacteraemia score. The first possible reason may be the poor performance of the Pitt bacteraemia score $^{24}$ in our study, which is likely due to the low number of bacteraemic patients with high scores. Second, it may be that NP colonisation density correlates mostly with the presence of bacteraemia, but among bacteraemic patients provides little additional prognostic information. However, in the multivariable analysis, colonisation density rather than the presence of bacteraemia correlated with mortality. Even though the magnitude of the association between NP colonisation density and prognosis was low, these data are in concordance with pneumococcal load data from invasive specimens as indicated

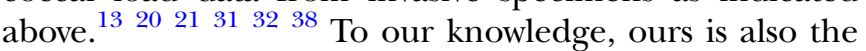
first study to assess systematically a large set of prognostic inflammatory and cardiovascular biomarkers among African HIV-infected adults with CAP. ProADM was the best prognostic biomarker, as previously shown in settings in the developed world, in largely HIV-uninfected adults. ${ }^{43}$ Since the peptides of the calcitonin gene family including ProADM and PCT as "hormokines" are ubiquitously upregulated in parenchymatous body cells and do not mainly originate from leucocytes, ${ }^{45}$ it is not unexpected that their prognostic quality can be extended to HIV-infected adults.
Correlation with a variety of severity markers with the presence of bacteraemia and with mortality all support $\mathrm{NP}$ colonisation density as a prognostic tool in HIV-infected adults with SP-CAP. Persistence or increase of bacterial loads over time by serial monitoring might indicate insufficient treatment, antibiotic resistance or insufficient focus control, while documented decreases of the bacterial load could allow discontinuation of antibiotic therapy. Waterer and Rello ${ }^{46}$ suggested that a high organism burden could indicate a risk for antibiotic-induced bacterial lysis, cytokine release and an unfavourable proinflammatory state with subsequent haemodynamic instability, which might benefit from immunomodulation. Therefore, a high colonisation density might be an indication for combination treatment with macrolides or other antibiotics that downregulate pneumolysin production, ${ }^{47}$ or for alternative anti-inflammatory treatments.

A limitation of our study includes the lack of data, particularly arterial blood gas analysis, to calculate the PSI. Owing to the setting, very few patients were admitted to ICU, which unfortunately makes this a not useful outcome measure. As there were few HIV-uninfected patients in our study, our results should be confirmed in a larger study of HIV-uninfected adults even though similar trends were found regardless of HIV status in our study (data not shown).

\section{CONCLUSIONS}

As previously reported, the quantitative lytA rtPCR from NP swabs is a very promising tool to diagnose pneumococcal pneumonia. ${ }^{22}$ In addition, this study shows that the same assay also conveys some prognostic information as it correlated with bacteraemia, survival and prognostic biomarkers. How exactly it could be implemented and how it might change management, such as site of care, antibiotic choices and duration, needs to be determined.

\section{Author affiliations}

${ }^{1}$ Division of Infectious Diseases and Hospital Epidemiology, Kantonsspital St Gallen, St Gallen, Switzerland

${ }^{2}$ Medical Research Council: Respiratory and Meningeal Pathogens Research Unit, Faculty of Health Sciences, Chris Hani Baragwanath Hospital, University of the Witwatersrand, Johannesburg, South Africa

${ }^{3}$ Department of Science and Technology/National Research Foundation, Vaccine Preventable Diseases University of the Witwatersrand, Johannesburg, South Africa

${ }^{4}$ Emerging Pathogens Laboratory, Fondation Mérieux, Lyon, France

${ }^{5}$ Thermo Scientific Biomarkers, Hennigsdorf, Germany

${ }^{6}$ Centre Pasteur du Cameroun, Yaoundé, Cameroun

${ }^{7}$ Department of Internal Medicine, Medical University Department, Kantonsspital Aarau, Aarau, Switzerland

${ }^{8}$ Hubert Department of Global Health and Division of Infectious Diseases,

Emory University, Atlanta, Georgia, USA

${ }^{9}$ Pneumonia Program, Bill and Melinda Gates Foundation, Seattle, Washington, USA

Contributors WCA had the idea, managed the study, collected the data, performed analyses, interpreted the data, drafted the manuscript and takes full responsibility of the overall content. SAM and KPK had the idea, interpreted 
the data and drafted the manuscript. NvN managed the study, was responsible for and performed the assays and collected the data. NE managed the study and collected the data. PVA and JNT were responsible for assays and interpreted the data. MM was responsible for and performed the assays and interpreted the data. GPB and SG were responsible for assays and interpreted the data. GV and BM interpreted the data. All authors amended and commented on the final manuscript.

Funding This work was funded by the Center for AIDS Research/NIH Grant P30 A1050409 to KPK; C-polysaccharide Antigen was provided free of charge by Binax; PCT sensitive LIA, MR-proADM KRYPTOR, MR-proANP KRYPTOR, Copeptin KRYPTOR were provided free of charge by Thermo Scientific Biomarkers, Hennigsdorf, Germany.

Competing interests WCA received an honorarium from GlaxoSmithKline (GSK) and support from BRAHMS Thermo Fisher and bioMérieux to attend meetings and fulfilled speaking engagements. SAM received research funding and honoraria from Pfizer vaccines and GSK and institutional grant support from Wyeth. KPK received consulting and research funding from Pfizer Vaccines and consulting funding from GSK. BM received support from BRAHMS and bioMérieux to attend meetings and fulfilled speaking engagements and research support from BRAHMS Thermo Fisher. SG is an employee of Thermo Scientific Biomarkers.

Ethics approval The study was approved by the ethics committees of the University of the Witwatersrand and Emory University.

Provenance and peer review Not commissioned; externally peer reviewed.

Data sharing statement Extra data can be accessed via the Dryad data repository at http://datadryad.org/ with the doi:10.5061/dryad.rr42f.

Open Access This is an Open Access article distributed in accordance with the Creative Commons Attribution Non Commercial (CC BY-NC 4.0) license, which permits others to distribute, remix, adapt, build upon this work noncommercially, and license their derivative works on different terms, provided the original work is properly cited and the use is non-commercial. See: http:// creativecommons.org/licenses/by-nc/4.0/

\section{REFERENCES}

1. Mortality and causes of death in South Africa, 2011: findings from death notification: statistics South Africa, 2014. http://beta2.statssa. gov.za/ (accessed 26 Jun 2014).

2. Woodhead M, Blasi F, Ewig S, et al. Guidelines for the management of adult lower respiratory tract infections-full version. Clin Microbiol Infect 2011;17(Suppl 6):E1-59.

3. Scott JA, Hall AJ, Muyodi C, et al. Aetiology, outcome, and risk factors for mortality among adults with acute pneumonia in Kenya. Lancet 2000;355:1225-30.

4. Jokinen J, Scott JA. Estimating the proportion of pneumonia attributable to pneumococcus in Kenyan adults: latent class analysis. Epidemiology 2010;21:719-25.

5. Feldman C, Klugman KP, Yu VL, et al. Bacteraemic pneumococcal pneumonia: impact of HIV on clinical presentation and outcome. $J$ Infect 2007;55:125-35.

6. Klugman KP, Madhi SA, Feldman C. HIV and pneumococcal disease. Curr Opin Infect Dis 2007;20:11-15.

7. Gilks CF, Ojoo SA, Ojoo JC, et al. Invasive pneumococcal disease in a cohort of predominantly HIV-1 infected female sex-workers in Nairobi, Kenya. Lancet 1996;347:718-23.

8. Mwachari CW, Nduba VN, Park DR, et al. Clinical presentation among HIV-infected and non-infected adults with community acquired pneumonia in Nairobi. Int $J$ Tuberc Lung Dis 2006;10:516-22.

9. Feikin DR, Feldman C, Schuchat A, et al. Global strategies to prevent bacterial pneumonia in adults with HIV disease. Lancet Infect Dis 2004;4:445-55.

10. Feikin DR, Schuchat A, Kolczak M, et al. Mortality from invasive pneumococcal pneumonia in the era of antibiotic resistance, 1995-1997. Am J Public Health 2000:90:223-9.

11. Song JH, Jung SI, Ki HK, et al. Clinical outcomes of pneumococcal pneumonia caused by antibiotic-resistant strains in asian countries: a study by the Asian Network for Surveillance of Resistant Pathogens. Clin Infect Dis 2004;38:1570-8.
12. Aspa J, Rajas O, Rodriguez de Castro F, et al. Impact of initial antibiotic choice on mortality from pneumococcal pneumonia. Eur Respir J 2006;27:1010-19.

13. Rello J, Lisboa T, Lujan M, et al. Severity of pneumococcal pneumonia associated with genomic bacterial load. Chest 2009;136:832-40.

14. Sandvall B, Rueda AM, Musher DM. Long-term survival following pneumococcal pneumonia. Clin Infect Dis 2013;56:1145-6.

15. Austrian R, Gold J. Pneumococcal bacteremia with especial reference to bacteremic pneumococcal pneumonia. Ann Intern Med 1964;60:759-76.

16. Lim WS, van der Eerden MM, Laing R, et al. Defining community acquired pneumonia severity on presentation to hospital: an international derivation and validation study. Thorax 2003;58:377-82.

17. Fine MJ, Auble TE, Yealy DM, et al. A prediction rule to identify low-risk patients with community-acquired pneumonia. $N$ Engl J Med 1997;336:243-50.

18. Schuetz $P$, Wolbers $M$, Christ-Crain M, et al. Prohormones for prediction of adverse medical outcome in community-acquired pneumonia and lower respiratory tract infections. Crit Care 2010;14: R106.

19. Albrich WC, Dusemund F, Ruegger K, et al. Enhancement of CURB65 score with proadrenomedullin (CURB65-A) for outcome prediction in lower respiratory tract infections: derivation of a clinical algorithm. BMC Infect Dis 2011;11:112.

20. Carrol ED, Guiver M, Nkhoma S, et al. High pneumococcal DNA loads are associated with mortality in Malawian children with invasive pneumococcal disease. Pediatr Infect Dis J 2007;26:416-22.

21. Peters RP, de Boer RF, Schuurman T, et al. Streptococcus pneumoniae DNA load in blood as a marker of infection in patients with community-acquired pneumonia. J Clin Microbiol 2009;47: 3308-12.

22. Albrich WC, Madhi SA, Adrian PV, et al. Use of a rapid test of pneumococcal colonization density to diagnose pneumococcal pneumonia. Clin Infect Dis 2012:54:601-9.

23. Chow JW, Fine MJ, Shlaes DM, et al. Enterobacter bacteremia: clinical features and emergence of antibiotic resistance during therapy. Ann Intern Med 1991;115:585-90.

24. Feldman C, Alanee S, Yu VL, et al. Severity of illness scoring systems in patients with bacteraemic pneumococcal pneumonia: implications for the intensive care unit care. Clin Microbiol Infect 2009;15:850-7.

25. Greenberg D, Givon-Lavi N, Newman N, et al. Nasopharyngea carriage of individual Streptococcus pneumoniae serotypes during pediatric pneumonia as a means to estimate serotype disease potential. Pediatr Infect Dis J 2011;30:227-33.

26. Christie IM. Bacteraemia in lobar pneumonia. Lancet 1933;2:804-5.

27. Landsman JB. Bacteriaemia and prognosis in lobar pneumonia: the results of quantitative blood culture in pneumococcus pneumonia. Glasgow Med J 1952;33:33-45.

28. Peralta G, Rodriguez-Lera MJ, Garrido JC, et al. Time to positivity in blood cultures of adults with Streptococcus pneumoniae bacteremia. BMC Infect Dis 2006;6:79.

29. Wolter N, Cohen C, Tempia S, et al. HIV and influenza virus infections are associated with increased blood pneumococcal load: a prospective, hospital-based observational study in South Africa, 2009-2011. J Infect Dis 2014;209:56-65.

30. Carvalho MD, Tondella ML, McCaustland K, et al. Evaluation and improvement of real-time PCR detection assays to lytA, ply, and psaA genes for detection of pneumococcal DNA. J Clin Microbio 2007;45:2460-6.

31. Munoz-Almagro C, Gala S, et al. DNA bacterial load in children and adolescents with pneumococcal pneumonia and empyema. Eur $J$ Clin Microbiol Infect Dis 2011;30:327-35.

32. Esposito S, Zampiero A, Terranova L, Selva L, et al. Pneumococcal bacterial load colonization as a marker of mixed infection in children with alveolar community-acquired pneumonia and respiratory syncytial virus or rhinovirus infection. Pediatr Infect Dis $J$ 2013;32:1199-204.

33. Said MA, Johnson HL, Nonyane BA, et al. Estimating the burden of pneumococcal pneumonia among adults: a systematic review and meta-analysis of diagnostic techniques. PLOS ONE 2013;8:e60273.

34. Feldman C, Anderson R. Bacteraemic pneumococcal pneumonia: current therapeutic options. Drugs 2011;71:131-53.

35. Resti M, Moriondo M, Cortimiglia M, et al. Community-acquired bacteremic pneumococcal pneumonia in children: diagnosis and serotyping by real-time polymerase chain reaction using blood samples. Clin Infect Dis 2010;51:1042-9.

36. Grijalva CG, Nuorti JP, Arbogast PG, et al. Decline in pneumonia admissions after routine childhood immunisation with pneumococca 
conjugate vaccine in the USA: a time-series analysis. Lancet 2007;369:1179-86.

37. Musher DM, Montoya R, Wanahita A. Diagnostic value of microscopic examination of Gram-stained sputum and sputum cultures in patients with bacteremic pneumococcal pneumonia. Clin Infect Dis 2004;39:165-9.

38. Werno AM, Anderson TP, Murdoch DR. Association between pneumococcal load and disease severity in adults with pneumonia. J Med Microbiol 2012;61(Pt 8):1129-35.

39. Olfert J, Skinner S. Validity of CURB-65 in predicting mortality and ICU admission in HIV-positive patients with community-acquired pneumonia. CHEST 2012 Annual Meeting. Atlanta, GA: CHEST, 2012:189A.

40. Martin GE, Gouillou M, Hearps AC, et al. Age-associated changes in monocyte and innate immune activation markers occur more rapidly in HIV infected women. PLOS ONE 2013;8:e55279.

41. Hearps AC, Maisa A, Cheng WJ, et al. HIV infection induces age-related changes to monocytes and innate immune activation in young men that persist despite combination antiretroviral therapy. AIDS 2012;26:843-53.
42. Pathai S, Lawn SD, Gilbert CE, et al. Accelerated biological ageing in HIV-infected individuals in South Africa: a case-control study. AIDS 2013;27:2375-84.

43. Schuetz P, Litke A, Albrich WC, et al. Blood biomarkers for personalized treatment and patient management decisions in community-acquired pneumonia. Curr Opin Infect Dis 2013;26:159-67.

44. Huang DT, Angus DC, Kellum JA, et al. Midregional proadrenomedullin as a prognostic tool in community-acquired pneumonia. Chest 2009:136:823-31.

45. Linscheid P, Seboek D, Zulewski $\mathrm{H}$, et al. Autocrine/paracrine role of inflammation-mediated calcitonin gene-related peptide and adrenomedullin expression in human adipose tissue. Endocrinology 2005;146:2699-708.

46. Waterer G, Rello J. Why should we measure bacterial load when treating community-acquired pneumonia? Curr Opin Infect Dis 2011;24:137-41.

47. Anderson R, Steel HC, Cockeran R, et al. Clarithromycin alone and in combination with ceftriaxone inhibits the production of pneumolysin by both macrolide-susceptible and macrolide-resistant strains of

Streptococcus pneumoniae. J Antimicrob Chemother 2007:59:224-9. 